\title{
No Acceleration of Intervertebral Disc Degeneration after a Single Injection of Bupivacaine in Young Age Group with Follow-Up of 5 Years
}

\author{
Seiji Ohtori, Gen Inoue, Sumihisa Orita, Yawara Eguchi, Nobuyasu Ochiai, Shunji Kishida, \\ Kazuki Kuniyoshi, Junichi Nakamura, Yasuchika Aoki, Tetsuhiro Ishikawa, Masayuki Miyagi, \\ Hiroto Kamoda, Miyako Suzuki, Masashi Takaso, Tomoaki Toyone, Gou Kubota, \\ Yoshihiro Sakuma, Yasuhiro Oikawa, Kazuhide Inage, Takeshi Sainoh, Kazuhisa Takahashi
}

Department of Orthopaedic Surgery, Graduate School of Medicine, Chiba University, Chiba, Japan

\begin{abstract}
Study Design: Prospective study of changes in intervertebral disc degeneration after injection of bupivacaine.
Purpose: To examine whether injection of bupivacaine into human intervertebral discs accelerates their degeneration.

Overview of Literature: Bupivacaine is commonly used for therapy and diagnosis of discogenic low back pain. However, several in vitro studies have reported toxic effects of bupivacaine to disc cells. We sought to evaluate whether this finding is clinically relevant. Methods: We selected 46 patients with low back pain who showed disc degeneration at only one level (L4-L5 or L5-S1) on magnetic resonance imaging (MRI) (discography group, $n=18$ ), discoblock group (injection of bupivacaine, $n=18$ ), and a control group, $n=10$ ). There were no significant differences in baseline characteristics across the 3 groups. The two experimental groups underwent either discography or anesthetic discoblock, respectively. All three groups were followed up 5 years after the examination.

Results: At 5 years follow-up, there was no significant difference in the rate of disc degeneration among the 3 groups (p>0.1). Moreover, $\mathrm{X}$-ray images showed that there was no significant difference in disc height, range of motion, or translation between flex and extension position $(p>0.1)$.

Conclusions: In conclusion, radiologic and MRI findings did not show acceleration of intervertebral disc degeneration at 5 years after a single injection of bupivacaine into human discs.
\end{abstract}

Keywords: Lumbar vertebrae; Intervertebral disc degeneration; Bupivacaine

\section{Introduction}

Low back pain (LBP) is a common clinical problem and is of major socioeconomic importance. However, little is known about the pathogenesis of this disease. Although any of the spinal structures (intervertebral discs, facet joints, vertebral bodies, ligaments, or muscles) may con- tribute to LBP, the most likely cause is a lumbar intervertebral disc [1-3].

Bupivacaine is commonly used for therapy and diagnosis of discogenic low back pain. Moreover, bupivacaine has been injected in combination with steroids, oxygen, ozone, and hypertonic dextrose, for the treatment of discrelated pain $[4,5]$.

Received Jul 19, 2012; Revised Aug 14, 2012; Accepted Aug 17, 2012

Corresponding author: Seiji Ohtori

Department of Orthopaedic Surgery, Graduate School of Medicine, Chiba University,

1-8-1 Inohana, Chuo-ku, Chiba 260-8670, Japan

Tel: +81-43-226-2117, Fax: +81-43-226-2116, E-mail: sohtori@faculty.chiba-u.jp 
Several authors have reported a toxic effect of bupivacaine on articular chondrocytes. It has been reported that $0.5 \%$ bupivacaine, which is commonly used in intra-articular surgery, has a toxic effect on isolated bovine articular chondrocytes [6]. Effects of bupivacaine on rabbit knee joints have been investigated, and compared to a placebo injection with saline, intra-articular bupivacaine injection had caused histopathological changes in rabbit knee joints [7]. In rabbit shoulder, infusion of bupivacaine decreased relative cell viability by $20 \%$ to $32 \%$. Histological analysis had yielded results significantly worse for bupivacaine infusion [8]. Thus, intra-articular infusion of bupivacaine was believed to cause significant histopathological and metabolic changes to articular cartilage [8].

Lee et al. [9] recently reported an in vitro controlled study on the effect of bupivacaine on viability of human and rabbit intervertebral disc cells. The results of this study has shown that bupivacaine decreases viability in rabbit and human disc cells in a time-dependent manner. Moreover, the changes observed for these disc cells were greater than those seen for articular chondrocytes. However, there has not been any report on whether these animal and in vitro studies are clinically relevant. Thus, the purpose of this current study was to examine whether injection of bupivacaine into human intervertebral disc accelerated discdegeneration.

\section{Materials and Methods}

The ethics committee of our institution approved the study design. Written informed written consent was obtained from each subject prior to participation in the study.

\section{Patients}

We reviewed 82 patients who had LBP, continuing for at least 3 years. Disc degeneration at one level on magnetic resonance imaging (MRI) was observed in all patients. Patients who had severe spondylolysis or disc degeneration of two or multilevel lesions on MRI were excluded. Patients who had previously undergone spinal surgery were also excluded.

To investigate the origin of pain, each patient underwent discography or discoblock with injection of bupivacaine. If low back pain was provoked in the discography, and only if the pain ameliorated with discoblock, the di- agnosis of discogenic pain was confirmed. Thirty of these patients subsequently underwent anterior discectomy and anterior lumbar interbody fusion or posterolateral fusion and were excluded from the study.

The remaining 52 patients tested negative for provocation of pain by discography or for pain relief after discoblock, and underwent nonoperative treatment. Sixteen patients were dropped out, and finally 36 patients were evaluated and followed up. For the control group of the study, 10 patients were enrolled to match age, pain, and disc degeneration to the 36 patients in the experimental group. Finally, we divided the patients into 3 groups (discography, $\mathrm{n}=18$; discoblock [injection of bupivacaine], $\mathrm{n}=18$; and control, $\mathrm{n}=10$ ).

\section{X-ray imaging evaluation}

Disc height, translation between flex and extension position, and range of motion (ROM; disc angle between flex and extension position) were evaluated from profile views on X-ray films before and 5 years after bupivicane injection. Evaluation of these parameters were performed by three blinded observers. The average measurement from three observers was used.

\section{MRI evaluation}

The initial and five-year disc degeneration was graded by Pfirrmann's grading (from grade I to V) using MRI [10]. Evaluation was performed by same three observers as above. If a grading from two of the observers were in agreement, this classification was used to define the intervertebral disc degeneration. We defined Pfirrmann's grading to score (i.e., I was score $1, \mathrm{~V}$ was score 5). The average score of Pfirrmann's classification was calculated as follows:

Average score $=\Sigma$ each patient's score/number of patients.

Reliability assessment of MRI was determined by calculating Cronbach a coefficient, which normally ranges between 0 and 1 with a coefficient of 1.0 representing perfect interobserver and intraobserver reliability.

\section{Pain evaluation}

Before examination, the visual analogue scale (VAS) score ( 0 , no pain; 10 , worst pain), and oswestry disability index 
Table 1. Demographic characteristics

\begin{tabular}{|c|c|c|c|c|}
\hline Characteristic & Control & Discography & Discoblock & $\begin{array}{c}\text { Statistical } \\
\text { analysis } \\
\text { (p-value) }\end{array}$ \\
\hline No. of patients & 10 & 18 & 18 & - \\
\hline Sex (male:female) & $5: 5$ & $10: 8$ & $9: 9$ & - \\
\hline Age (yr) & $35 \pm 5(18-54)$ & $36 \pm 6(22-44)$ & $36 \pm 4(22-43)$ & 0.60 \\
\hline Symptom duration (yr) & $6(4-10)$ & $6(3-20)$ & $6(4-12)$ & 0.66 \\
\hline Follow-up (yr) & $4(3-5)$ & $3.5(3-4)$ & $3.6(3-4)$ & 0.50 \\
\hline \multicolumn{5}{|l|}{ MRI findings } \\
\hline$\llcorner 4 / 5$ & 7 & 10 & 11 & - \\
\hline L5/S1 & 3 & 8 & 7 & - \\
\hline \multicolumn{5}{|l|}{ Pfirrmann's classification } \\
\hline I & 0 & 0 & 0 & - \\
\hline I & 0 & 0 & 0 & - \\
\hline III (L4/5, L5/S1) & $3(2,1)$ & $6(2,4)$ & $7(4,3)$ & - \\
\hline IV (L4/5, L5/S1) & $7(5,2)$ & $12(8,4)$ & $11(7,4)$ & - \\
\hline V & 0 & 0 & 0 & - \\
\hline Average & $3.7 \pm 0.48$ & $3.8 \pm 0.42$ & $3.7 \pm 0.48$ & $>0.1$ \\
\hline \multicolumn{5}{|l|}{ Pain score } \\
\hline Visual analogue scale leg pain & $3.0 \pm 1.5$ & $3.6 \pm 1.4$ & $2.5 \pm 1.5$ & 0.58 \\
\hline Visual analogue scale low back pain & $5.8 \pm 1.9$ & $7.4 \pm 2.3$ & $7.2 \pm 2.8$ & 0.60 \\
\hline Oswestry Disability Index & $52 \pm 10$ & $66 \pm 16$ & $70 \pm 18$ & 0.52 \\
\hline
\end{tabular}

Values are presented as mean \pm standard error mean (range) or mean (range).

(ODI) were recorded and compared across the 3 groups.

\section{Discography and discoblock for a degenerated disc at a single level}

We conducted an examination of discography or discoblock for diagnosis of discogenic LBP. Discography and discoblock were performed with a standard posterolateral approach using a 22-gauge needle (Becton Dickinson, Franklin Lakes, NJ, USA). The needle was inserted into the center of the disc under fluoroscopic control. For discography, Isovist $240 \mathrm{~mL}$ (range, 0.4-2.0 mL; Schering, Berlin, Germany) was injected into each disc until pain was provoked. We did not perform a control provocative discogram in the adjacent segments.

Bupivacaine $(0.75 \mathrm{~mL}$ of $0.5 \%)$ was injected into the disc in the same manner as for the discoblock. For the 10 patients in the control group, neither of these examinations were performed.

\section{Statistical analysis}

Data were compared using a Kruskal-Wallis test to compare pain scales across the three groups (Table 1). A one way analysis of variance with post hoc comparisons was used for age, symptom duration (Table 1), and X-ray and MRI evaluations (Tables 2-4). Fisher's test was used for dichotomous/categorical variables (Table 4). $p<0.05$ was considered statistically significant.

\section{Results}

\section{Patient background}

Table 1 shows the baseline characteristics of patients. There were no statistically significant differences in sex, age, symptom duration, period of follow-up, or pain score in either group.

The interobserver and intraobserver reliability scores for MRI evaluations were excellent with calculated Cron- 
Table 2. Evaluation of instability before examination

\begin{tabular}{|c|c|c|c|c|}
\hline Characteristic & Control & Discography & Discoblock & $\begin{array}{c}\text { Statistical analysis } \\
\text { (p-value) }\end{array}$ \\
\hline \multicolumn{5}{|l|}{$\llcorner 4 / 5$} \\
\hline Disc height (mm) & $8.2 \pm 1.3$ & $9.5 \pm 2.0$ & $8.7 \pm 1.7$ & 0.50 \\
\hline Translation (mm) & $2.2 \pm 1.2$ & $2.5 \pm 1.4$ & $2.8 \pm 1.0$ & 0.41 \\
\hline Range of motion $\left({ }^{\circ}\right)$ & $8.2 \pm 2.1$ & $9.2 \pm 2.3$ & $8.5 \pm 2.0$ & 0.56 \\
\hline \multicolumn{5}{|l|}{$\mathrm{L} 5 / \mathrm{S} 1$} \\
\hline Disc height (mm) & $5.2 \pm 1.1$ & $5.5 \pm 1.0$ & $5.8 \pm 1.9$ & 0.60 \\
\hline Translation (mm) & $2.0 \pm 1.0$ & $2.2 \pm 1.2$ & $2.4 \pm 1.2$ & 0.38 \\
\hline Range of motion $\left({ }^{\circ}\right)$ & $6.5 \pm 2.2$ & $6.8 \pm 1.4$ & $5.7 \pm 1.9$ & 0.58 \\
\hline
\end{tabular}

Table 3. Evaluation of instability 5 years after examination

\begin{tabular}{|c|c|c|c|c|}
\hline Characteristic & Control & Discography & Discoblock & $\begin{array}{c}\text { Statistical analysis } \\
\text { (p-value) }\end{array}$ \\
\hline \multicolumn{5}{|l|}{$L 4 / 5$} \\
\hline Disc height (mm) & $6.2 \pm 1.2$ & $7.8 \pm 2.1$ & $7.7 \pm 1.8$ & 0.20 \\
\hline Translation (mm) & $2.2 \pm 1.1$ & $2.3 \pm 1.2$ & $2.4 \pm 1.1$ & 0.26 \\
\hline Range of motion $\left({ }^{\circ}\right)$ & $7.2 \pm 2.2$ & $7.8 \pm 2.1$ & $7.6 \pm 2.1$ & 0.40 \\
\hline \multicolumn{5}{|l|}{$\mathrm{L} 5 / \mathrm{S} 1$} \\
\hline Disc height (mm) & $4.8 \pm 1.0$ & $4.9 \pm 1.1$ & $5.0 \pm 1.7$ & 0.30 \\
\hline Translation (mm) & $2.0 \pm 1.0$ & $2.0 \pm 1.1$ & $2.2 \pm 1.1$ & 0.33 \\
\hline Range of motion $\left({ }^{\circ}\right)$ & $6.0 \pm 2.0$ & $6.0 \pm 1.4$ & $5.6 \pm 1.5$ & 0.41 \\
\hline
\end{tabular}

bach a coefficients of 0.86 (before examination) and 0.90 (5 years after examination).

Across the 3 groups, there were no significant differences in disc degeneration $(p>0.1)$ and in the average score based on Pfirrmann's classification $(p>0.1)$ (Table 1). $\mathrm{X}$-ray imaging findings revealed no significant baseline differences of disc height, translation, and ROM at L4-L5 and L5-S1 ( $p>0.05)$ (Table 2).

\section{Changes in radiographic and MRI findings 5 years after examination}

At 5 years after the initial examination, disc height, translation, and ROM had decreased for subjects in all three groups (Tables 2, 3). However, the changes in these X-ray findings were consistent across all three groups $(p>0.05)$. Likewise, disc degeneration were identified for all of the subjects during the 5 years since the initial MRI evaluation. However, the rate of degeneration was not different across the three groups $(p>0.1)$. No significant differences were identified for the scores based on Pfirrmann's classification $(p>0.1)$ (Table 4$)$.

\section{Discussion}

In the current study, we evaluated disc degeneration over a five year period in patients who had received either discography or discoblock (injection of bupivacaine) and compared the results with control subject. Compared to the control or discography group, bupivacaine injection group did not show accelerated disc degeneration. We concluded that there was no toxic effect of bupivacaine to intervertebral disc based on radiographic and MRI study.

Bupivacaine has been found to be chondrotoxic in vitro, and several studies have shown a dose- and timedependent chondrotoxicity of local anesthetics [6-8]. Furthermore, an effect of $0.5 \%$ bupivacaine on disc cells and articular chondrocytes in vitro has been reported [9]. Rabbit and human disc cell death demonstrated a time and dose dependence in response to bupivacaine [9]. 
Table 4. Change in magnetic resonance imaging findings

\begin{tabular}{lcr} 
Pfirrmann's classification & Before & After \\
Control & & \\
\hline III $(L 4 / 5, L 5 / S 1)$ & $3(2,1)$ & $1(1,0)$ \\
\hline IV $(L 4 / 5, L 5 / S 1)$ & $7(5,2)$ & $8(6,2)$ \\
V & - & $1(0,1)$ \\
Average & $3.7 \pm 0.48$ & $4.0 \pm 0.48^{\text {a) }}$ \\
Discography & & \\
\hline III (L4/5, L5/S1) & $6(2,4)$ & $3(1,2)$ \\
\hline IV (L4/5, L5/S1) & $12(8,4)$ & $10(8,2)$ \\
\hline V & - & $5(2,3)$ \\
\hline Average & $3.8 \pm 0.42$ & $4.1 \pm 0.54^{a)}$ \\
\hline Discoblock & & \\
\hline III (L4/5, L5/S1) & $7(4,3)$ & $3(1,2)$ \\
\hline IV (L4/5, L5/S1) & $11(7,4)$ & $11(9,2)$ \\
\hline V & - & $4(2,2)$ \\
\hline Average & $3.7 \pm 0.48$ & $4.0 \pm 0.48^{a)}$ \\
\hline a) $p>0.1$. & & \\
\hline
\end{tabular}

Moreover, cell death was greater than that observed for articular chondrocytes [9]. These reports indicated that $0.5 \%$ bupivacaine had an apparent toxic effect on disc cells and articular chondrocyte of animals and humans in vitro [6-9]. Wang et al. [11] also reported an in vitro study that harmful effects of bupivacaine on several cell types, including nucleus pulposus and annulus fibrosus cells, both in cell and disc organ models. And bupivacaine negatively impacts disc cell viability and matrix metabolism, both of which strongly correlate to intervertebral disc degeneration.

An effect of $0.5 \%$ bupivacaine on articular chondrocytes in vivo has been reported; however, the effect was somewhat different from data obtained by in vitro studies [12]. Rats received a $0.5 \%$ bupivacaine into the knee joint and were observed during 6 months. In vivo study showed a significant reduction in chondrocyte density 6 months after a single intra-articular injection of $0.5 \%$ bupivacaine. This result was in agreement with in vitro study of rat cells [12]. However, the articular surfaces of bupivacaine-injected joints remained intact on gross and histological evaluation. The authors of that study concluded that potential toxic effects of bupivacaine may exist following a single injection, but that this would be difficult to detect clinically [12].

In this study, there was no significant difference in disc degeneration at 5 years following bupivacaine injection according to the evaluation using Pfirrmann's classification. Furthermore, there was no significant difference in disc height, range of motion, or translation between flex and extension position. There is a possibility that cell death and toxic effects had occurred in the disc of the patients who had received discoblock; however, this change does not appear to be clinically and radiographically relevant. Further study is needed to clarify the toxic effect using high sensitivity MRI or biopsy.

Additionally, there was no significant difference in progression of disc degeneration between control and discography groups at 5 year follow-up. Carragee et al. [13] recently reported that modern discography techniques using small gauge needles and limited pressurization resulted in accelerated disc degeneration, disc herniation, loss of disc height and signal, and the development of reactive endplate changes compared with matched-controls at 10-year follow-up [13]. This apparent difference may be because of differences in the number of patients and follow-up periods. Further longer-term follow-up is needed to clarify this point.

This study has several limitations. First of all, we have examined a small number of patients in each group. Second, the average age of the patients was around 35-yearold, however, the average of disc degeneration was from 3.7 to 3.8 before examination. grade $\mathrm{V}$ change has occurred in 10 patents in all patient 5 years after examination. In this study, the patient's population tended to show more severe degeneration of disc. Finally, a single or multiple injections of bupivacaine, the volume of bupivacaine, concentration, degenerative stage of the disc to be injected, and how the injection was given were not examined in the current study. A follow-up study with a larger number of patients and dose-dependent measurements of bupivacaine is needed to validate the findings from this study.

\section{Conclusions}

In conclusion, bupivacaine does not appear to accelerate intervertebral disc degeneration in patients undergoing discoblock. We concluded that the effect of bupivacaine on discs is different between in vitro and clinical settings. 


\section{Conflict of Interest}

No potential conflict of interest relevant to this article was reported.

\section{References}

1. Nachemson AL. The lumbar spine an orthopaedic challenge. Spine 1976;1:59-71.

2. Mooney V. Presidential address. International Society for the Study of the Lumbar Spine. Dallas, 1986. Where is the pain coming from? Spine (Phila Pa 1976) 1987;12:754-9.

3. Deyo RA, Weinstein JN. Low back pain. N Engl J Med 2001;344:363-70.

4. Gallucci M, Limbucci N, Zugaro L, et al. Sciatica: treatment with intradiscal and intraforaminal injections of steroid and oxygen-ozone versus steroid only. Radiology 2007;242:907-13.

5. Miller MR, Mathews RS, Reeves KD. Treatment of painful advanced internal lumbar disc derangement with intradiscal injection of hypertonic dextrose. Pain Physician 2006;9:115-21.

6. Chu CR, Izzo NJ, Papas NE, Fu FH. In vitro exposure to $0.5 \%$ bupivacaine is cytotoxic to bovine articular chondrocytes. Arthroscopy 2006;22:693-9.

7. Dogan N, Erdem AF, Erman Z, Kizilkaya M. The effects of bupivacaine and neostigmine on articular cartilage and synovium in the rabbit knee joint. J Int Med Res 2004;32:513-9.

8. Gomoll AH, Kang RW, Williams JM, Bach BR, Cole BJ. Chondrolysis after continuous intra-articular bupivacaine infusion: an experimental model investigating chondrotoxicity in the rabbit shoulder. Arthroscopy 2006;22:813-9.

9. Lee H, Sowa G, Vo N, et al. Effect of bupivacaine on intervertebral disc cell viability. Spine J 2010;10:15966.

10. Pfirrmann CW, Metzdorf A, Zanetti M, Hodler J, Boos N. Magnetic resonance classification of lumbar intervertebral disc degeneration. Spine (Phila $\mathrm{Pa}$ 1976) 2001;26:1873-8.

11. Wang D, Vo NV, Sowa GA, et al. Bupivacaine decreases cell viability and matrix protein synthesis in an intervertebral disc organ model system. Spine J 2011;11:139-46.

12. Chu $\mathrm{CR}$, Coyle $\mathrm{CH}$, Chu $\mathrm{CT}$, et al. In vivo effects of single intra-articular injection of $0.5 \%$ bupivacaine on articular cartilage. J Bone Joint Surg Am 2010;92:599-608.

13. Carragee EJ, Don AS, Hurwitz EL, Cuellar JM, Carrino JA, Herzog R. 2009 ISSLS Prize Winner: does discography cause accelerated progression of degeneration changes in the lumbar disc: a ten-year matched cohort study. Spine (Phila Pa 1976) 2009;34:2338-45. 